\title{
Modelling of Dishing for Metal Chemical Mechanical Polishing
}

\author{
Viet H. Nguyen ${ }^{(1)}$, Peter van der Velden ${ }^{\left(2^{*}\right)}$, Roel Daamen ${ }^{(2)}$, Herma van Kranenburg ${ }^{(1)}$ and Pierre H. Woerlee ${ }^{(1,2)}$ \\ (1) MESA+ Research Institute, University of Twente, P.O. box 217, 7500 AE Enschede, the Netherlands \\ (2) Philips Research, Prof. Holstlaan 4, 5656 AA Eindhoven, the Netherlands
}

\begin{abstract}
In this paper, a physical model for the development of dishing during metal chemical mechanical polishing (CMP) is proposed. The main assumption of the model is that material removal occurs predominantly at the pad/wafer contacts. The distribution of pad/wafer contact size is studied first. This distribution is used as an input for a model of the dependence for the material removal rate on the line width. A relation that describes the development of dishing as a function of overpolish time will be presented. The model describes to a great accuracy the observed dishing effects, using one free parameter.
\end{abstract}

\section{Introduction}

Metal chemical mechanical polishing (CMP) has been recognized as the key technology for IC back-end processes. A major issue that remains is the dishing of metal lines. Dishing reduces the thickness of metal line causing an increase in line's resistance as well as in current density flowing through the line. Consequently, dishing reduces the speed of signal propagation through the circuit and threatens the reliability of metal line at high current density. In addition, dishing decreases the planarity of the wafer surface. This complicates the multi-level metallisation process [1]. Recently dishing has been categorized as a must-be-controlled parameter in the metal CMP process - see table 1 [2]. In this work, dishing during Copper CMP is studied and a physical model is proposed. The model accounts for the morphology and properties of the polishing pad and process parameters. One free parameter is needed to describe the experimental dishing data.

\section{Experimental details}

Copper CMP experiments were performed on a Mirra ${ }^{\mathrm{TM}}$ polishing tool. An IC1400 pad was used. Structured wafers were polished with different pressures and overpolishing times. The overpolish time was controlled by a state-of-theart end-point detector.

\section{Experimental results}

In figure 1 the blanket removal rate of Copper is plotted versus applied pressure. Preston's law [3] is followed. Further study showed that Preston's law is also valid at very low pressures. In figure 2 the time dependence of dishing is shown for various feature sizes. It appears that dishing occurs mainly during overpolishing. Small deviation may be due to chemical etching. In figure 10 dishing is plotted versus feature size for three overpolishing times. Clearly dishing increases strongly with feature size and saturates for larger line width. Furthermore a strong time dependence is observed for large line width.

\section{Model}

To understand the origin of dishing during metal CMP, a model based on contacts mechanics is developed. Because of the large number of pad/wafer contacts, statistical technique is used The following assumptions are made: 1) dishing occurs during overpolishing, 2) Preston's law is valid for all polish conditions, 3) material removal occurs at the mechanical contact between the pad asperities and wafer, 4) the distribution of pad asperity contact size is Gaussian, 5) a different removal rate (RR) occurs for asperities with contact size smaller (S) and larger (B) than the line width , 6) oxide erosion is neglected and 7) a homogeneous distribution of abrasive particles embedded into the pad surface. The model assumptions are reasonable for modem metal CMP processes and realistic overpolish time. In figure 3 a $3 \mathrm{D}$ surface plot of the polish pad is shown. The surface can be modelled well by assuming spherical asperities with a Gaussian size (R) and height $(H)$ distribution $\Phi_{R}\left(R_{a}, \sigma_{a}\right)$ and $\Phi_{H}\left(H_{a}, \sigma_{h}\right)$. Here $R_{a}$, $H_{a}, \sigma_{a}, \sigma_{h}$ are the median values and standard deviation of the asperity size and height distributions. Two other important parameters are the surface asperity density $\eta$ and the pad Young modulus E. Experimentally determined values for $R_{a}, \sigma_{a}, \sigma_{h}$ and $\eta$ are $30 \mu \mathrm{m}, 25 \mu \mathrm{m}, 10 \mu \mathrm{m}$ and $1.210^{5} \mathrm{~cm}^{-2}$. E is equal to $29 \mathrm{Mpa}[4] . \mathrm{Ha}$ is the reference level and is set for convenience to $0 \mu \mathrm{m}$. Contact of the pad and a single asperity are schematically shown in figure $4 a, b$. Contact properties of a single asperity i.e. the contact radius $r_{c}$ and load $l$ carried by the asperity can be obtained from the theory of Hertz $[5,6]$.

$r_{C}=\sqrt{R \cdot(H-G)}$

$l=4 / 3 \cdot E \sqrt{R \cdot(H-G)^{3}}$

Here $\mathrm{G}$ is the gap between the reference plane $\left(\mathrm{H}_{\mathrm{a}}=0 \mu \mathrm{m}\right)$ and the wafer surface. At a certain separation distance $G$ there will only be a few asperities, which have height $H$ larger than $G$, and are touching the wafer. The number and size distribution of these contacts can be determined using equations 1 and 2 .

In order to achieve a separation gap $G$ between the reference plane of the polishing pad and the wafer surface, a pressure $P$ must be applied to the wafer:

$P=\frac{L}{A}=\eta \cdot \int_{G}^{\infty} \int_{0}^{\infty} l \cdot \Phi(R) \Phi(H) d R d H$

where $\mathrm{L}$ is the total load carried by asperities within a pad area of A. Knowing the applied pressure P, morphology and properties of the polishing pad the separation gap $G$ can be calculated using a numerical method. Consequently the number of contact and contact size distribution can be determined. Figure 5 shows the flow-chart of the numerical method used to determine the number of contacts and the contact size distribution for a certain polish pressure and pad

* with Rodel Europe since October $1^{\text {st }} 2000$ 
morphology. Using the IC1400 pad information the number of contacts is found to be a linear function of the applied pressure as shown in figure 6 . Incorporation of this result in the Preston relation gives:

$$
R R_{B l}=K_{p} \cdot K_{n} \cdot N \cdot V
$$

where $R R_{B l}$ is the blanket removal rate, $K_{p}$ is the Preston constant, $\mathrm{V}$ is the linear velocity, $\mathrm{N}$ the total number of contacts and $K_{n}$ is a proportionality constant linking the number of contacts with the applied pressure (determined from fig. 6). About 1 percent of the asperities is touching the surface, the average separation is larger than the largest line dimension $(100 \mu \mathrm{m})$. At a pressure of $250 \mathrm{gr} / \mathrm{cm}^{2}$ the average contact radius and standard deviation are $8.2 \mu \mathrm{m}$ and $5.5 \mu \mathrm{m}$ respectively. Figures 7 and 8 show the contact size distribution as function of pressure, height distribution, radius and its standard deviation and Young's modulus. Clearly the pad morphology has the largest impact on the contact size distribution.

To model the dishing of a line with width LW, the material removal by asperities with contact diameter smaller than LW (S) and larger than LW (B) has to be considered separately (see fig. $4 c$ ). For group $S$ the removal rate $R R_{S}$ is similar to the blanket removal, however Preston's law has to be corrected for the lower number of asperities $\mathrm{N}_{s}$ with contact radius $\mathrm{r}_{\mathrm{c}}<\mathrm{LW} / 2$.

$$
\begin{aligned}
& R R_{S}=K_{p} \cdot K_{n} \cdot N_{S} \cdot V=K_{p} \cdot K_{n} \cdot N \cdot V \cdot \int_{0}^{L W / 2} \Phi\left(r_{C}\right) \cdot d r_{C} \\
& =K_{p} \cdot P \cdot V \cdot \int_{0}^{L W / 2} \Phi\left(r_{C}\right) \cdot d r_{C}
\end{aligned}
$$

where $\Phi\left(r_{C}\right)$ is the distribution of contact size; $r_{C a}$ and $\sigma_{C}$ are the average contact radius and standard deviation.

For group B with $r_{c}$ larger than $L W / 2$ the pressure that is exerted on the metal in the trench is geometry and time dependent. As illustrated in figure 9, the more dishing develops, the less pressure can be exerted on the metal in the trench because of the elasticity of asperity. According to theory of elasticity [7], the maximum deflection amount $d_{\max }$ of an asperity into a trench of trench-width $\mathrm{LW}$ under a pressure of $\mathrm{P}_{1}$ can be calculated as:

$$
d_{\max }=\frac{m \cdot\left(1-v^{2}\right) P_{1} \cdot L W}{E}
$$

where $v$ is Poisson ratio, $\mathrm{E}$ is Young modulus of the asperity i.e. of the pad material and $\mathrm{m}$ is a free numerical factor. Thus, the pressure P' exerted on the metal when a dishing amount Dish has been developed is

$$
P^{\prime}=P-P_{1}=P-\frac{E \cdot \text { Dish }}{m \cdot\left(1-v^{2}\right) \cdot L W}
$$

Obviously, at zero overpolish time when Dish $=0$ (i.e. assumption 1) $\mathrm{P}^{\prime}=\mathrm{P}$. The development of dishing amount by time is obtained using Preston law:

$$
\frac{d D i s h}{d t}=K_{p} \cdot V \cdot P^{\prime}
$$

Solving equations 7 and 8 for the time dependent of the pressure P' gives
$P^{\prime}=P \cdot \exp \left(-\frac{K_{p} \cdot V \cdot E}{m \cdot\left(1-v^{2}\right) \cdot L W} \cdot t\right)$

Substituting equation 9 into Preston equation, we obtain equation 10 for the removal rate $R_{B}$ of asperities group $B$ as function of the overpolish time $t$

$R R_{B}(t)=K_{p} \cdot P \cdot V \cdot \exp \left(\frac{-t}{L W \cdot K}\right) \int_{L W / 2}^{\infty} \Phi\left(r_{C}\right) \cdot d r_{C}$

where

$K=\frac{m \cdot\left(1-v^{2}\right)}{K_{p} \cdot V \cdot E}$

Adding the RR of group $S$ and $B$ (equations 5 and 10) we finally obtain equation 12 for the total time dependent of dishing amount for a line with a width $\mathrm{LW}$.

$\operatorname{Dish}(L W, t)=$

$R R_{B l} \cdot \int_{0}^{t}\left[\int_{0}^{L W / 2} \Phi\left(r_{C}\right) d r_{C}+\exp \left(\frac{-t}{L W \cdot K}\right) \cdot \int_{L W / 2}^{\infty} \Phi\left(r_{C}\right) d r_{C}\right] d t$

The only adjustable parameter is $\mathrm{K}$, other parameters can be obtained from the pad morphology, properties and the blanket removal rate $R_{B 1}$.

\section{Model verification and discussions}

In figure $10 \mathrm{a}, \mathrm{b}, \mathrm{c}$ experimental dishing data and predicted dishing data are plotted for three overpolishing times. Excellent agreement between the model and experimental data is obtained for realistic overpolishing times. Both time dependence and feature size dependence are modelled well. It is noteworthy that in this model erosion effect is neglected and therefore it only valid for a limited overpolishing time or for the process that have high selectivity in removal rate between metal and barrier/oxide materials. This can be seen in figure $10 \mathrm{c}$ that for a long overpolishing time erosion starts and actual dishing values are slightly smaller than those predicted by the model.

\section{Conclusions}

A physical model based on contacts mechanics for the development of dishing in metal CMP is developed. The model describes to a great accuracy the observed dishing effects. It can be used to predict, understand and control the amount of dishing in metal CMP.

\section{Acknowledgements}

Many thanks are due and sincerely given to Piet Bouten, Harry Nulens, Pascal Bancken and Greja Verheyden of Philips Research for their fruitful discussions and technical supports.

\section{References}

[1] Y. Morand, Microelectron. Eng., vol. 50, 2000, pp. 391 - 401

[2] International Technology Roadmap for Semiconductors, 1999, Interconnect.

[3] F.W. Preston, J. Soc. Glass Tech., vol. 11, 1927, pp. 214

[4] A. R. Baker, Proc. Electrochem. Soc. Meeting, vol. 96, 1996, pp. 228

[5] B.N.J. Persson, "Sliding friction: physical principles and applications", Springer, 1998, Chapter 5.

[6] T.K. Yu, C.C. Yu, M. Orlowski, IEDM Technical Digests, 1993, pp. 865.

[7] S. P. Timoshenko and J. N. Goodier, "Theory of Elasticity", $3^{\text {rd }}$ Ed. McGraw-Hill, 1970, Chapter 12. 
Table 1. Microprocessor interconnect technology requirements from [2].

\begin{tabular}{|c|c|c|c|c|c|c|}
\hline $\begin{array}{l}\text { Year } \\
\text { Technology node }(\mathrm{nm})\end{array}$ & $\begin{array}{l}1999 \\
180\end{array}$ & $\begin{array}{l}\mathbf{2 0 0 2} \\
130\end{array}$ & $\begin{array}{l}2005 \\
100\end{array}$ & $\begin{array}{l}\mathbf{2 0 0 8} \\
70 \\
\end{array}$ & $\begin{array}{l}2011 \\
50\end{array}$ & $\begin{array}{l}2014 \\
35\end{array}$ \\
\hline $\begin{array}{l}\mathrm{Cu} \text { local dishing }(\mathrm{nm}) \text {, } \\
5 \% \times \text { height }\end{array}$ & 18 & 14 & 11 & 9 & 7 & 5 \\
\hline $\begin{array}{l}\text { Cu intermediate dishing } \\
\text { (nm) } 15 \mu \mathrm{m} \text { wide wire, } \\
10 \% \times \text { height }\end{array}$ & 64 & 51 & 41 & 30 & 22 & 17 \\
\hline $\begin{array}{l}\text { Cu global wiring dish- } \\
\text { ing }(\mathrm{nm}) 15 \mu \mathrm{m} \text { wide } \\
\text { wire, } 10 \% \times \text { height }\end{array}$ & 116 & 95 & 76 & 55 & 38 & 29 \\
\hline
\end{tabular}

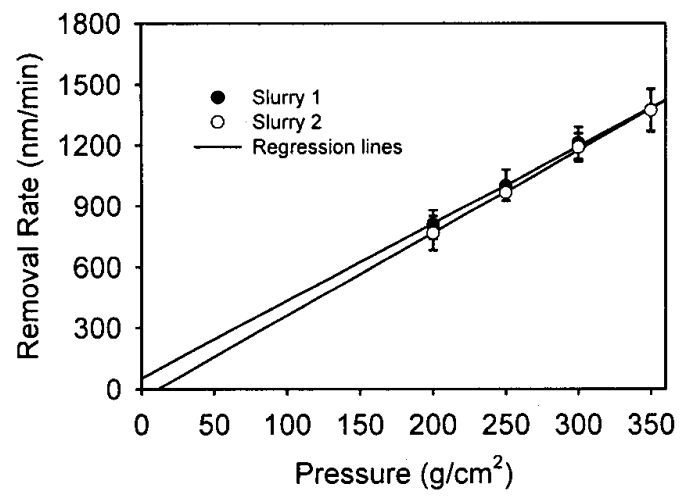

Fig. 1 Blanket removal rates of copper at different polishing pressures Slurry $1=$ QC1010: $\mathrm{H}_{2} \mathrm{O}_{2}=3: 1$ (vol.); Slurry $2=Q C 1010: \mathrm{H}_{2} \mathrm{O}_{2}=2: 1$ (vol.).

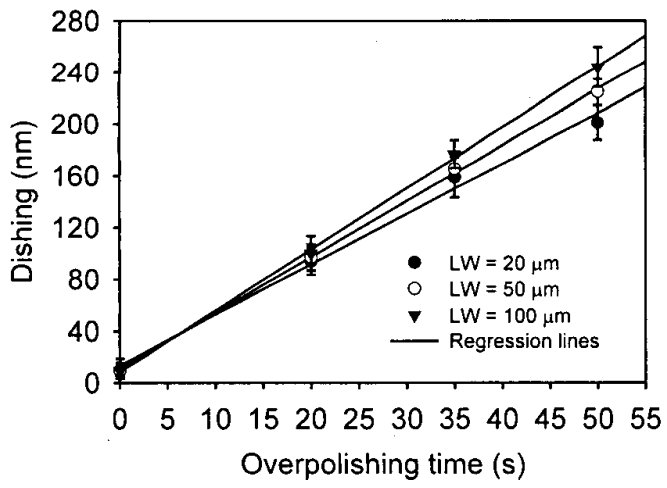

Fig. 2 Development of dishing as function of the overpolishing time.

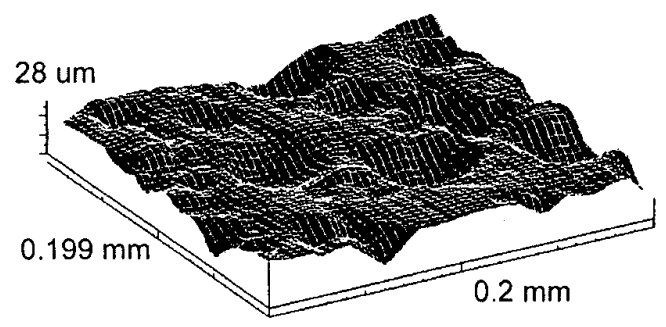

Fig. 3 Surface of an IC 1400 (Rodel) polishing pad after break-in procedure

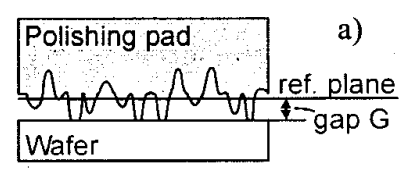

c)
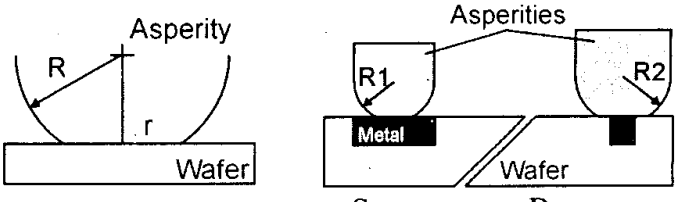

$\mathrm{S}$

B

Fig. 4 Schematic diagrams of the contact between polishing pad and wafer (a) contact between asperity and wafer (b) and contacts between asperities with relatively big and small trenches (c).

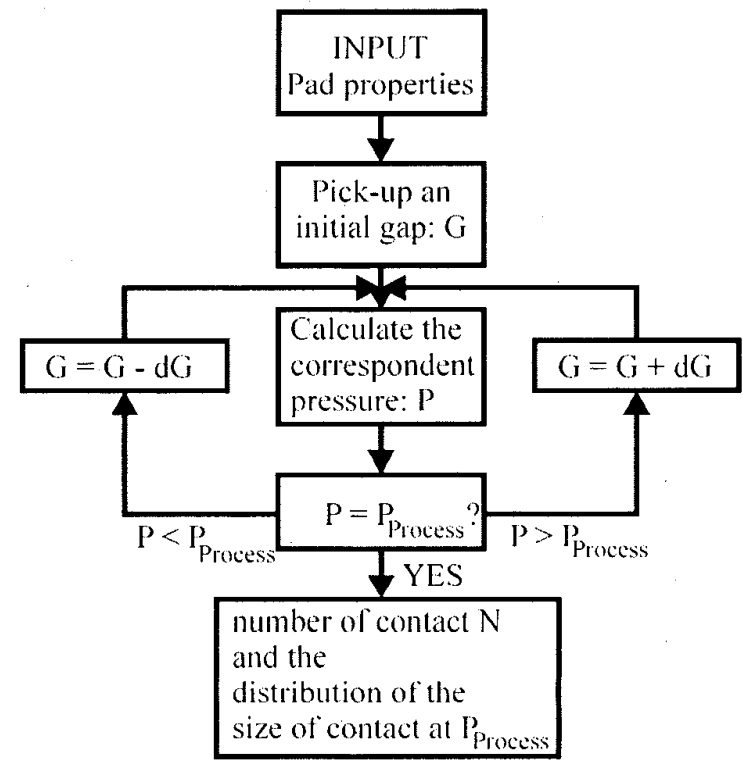

Fig. 5 Flow-chart diagram of the program that calculates the number of contacts and the distribution of the contact size.

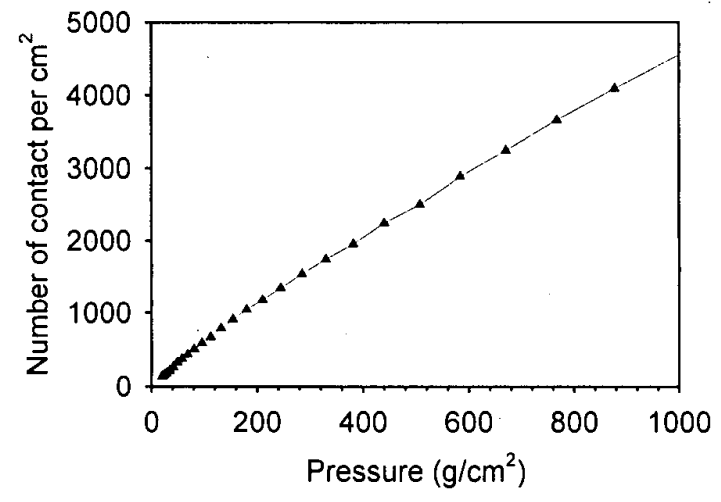

Fig. 6 Calculated number of contact versus applied pressure. 


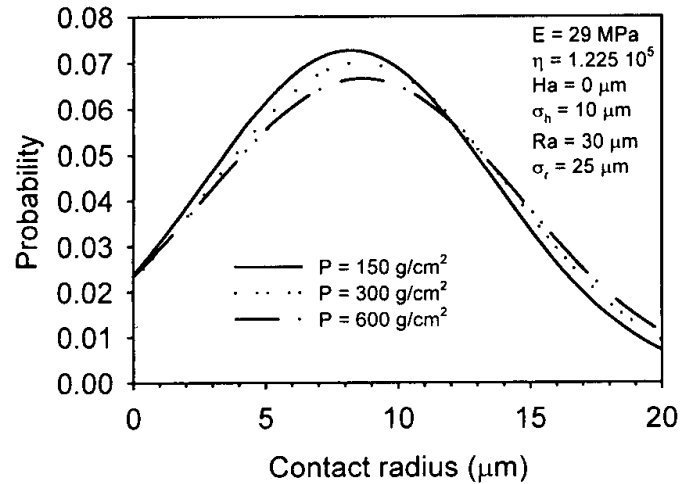

Fig. 7 Calculated contact size distribution at different pressures.
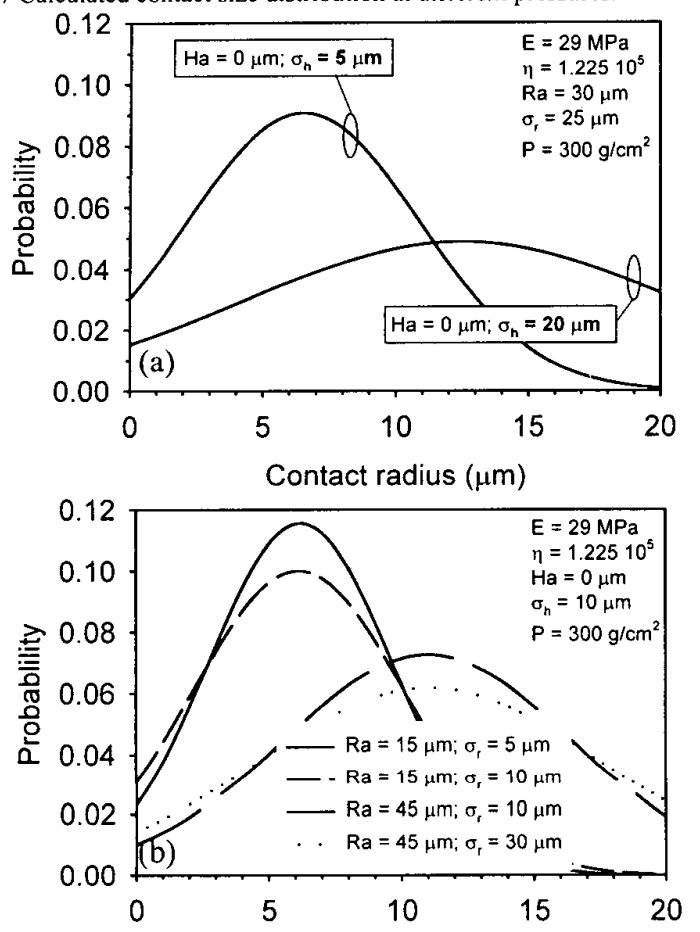

Contact radius $(\mu \mathrm{m})$

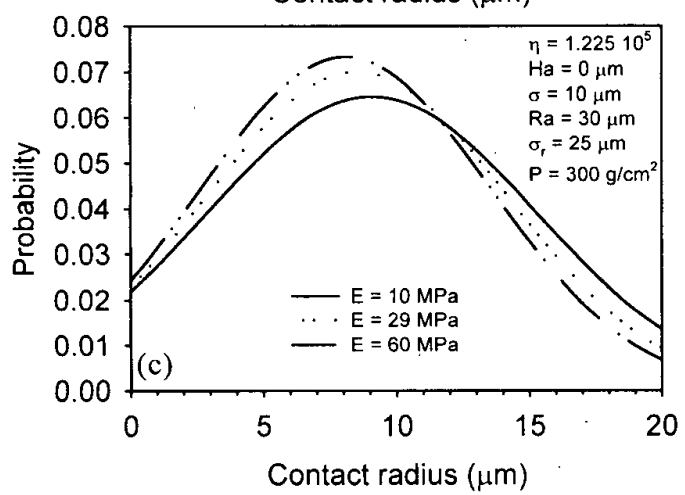

Fig. 8 Dependency of the contact size distribution on (a) the height distribution, (b) the size distribution of the asperities and (c) the Young modulus of the pad.

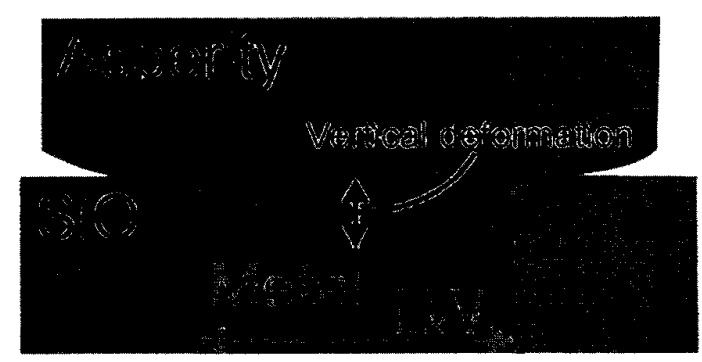

Fig. 9 Deformation of an asperity into a trench.
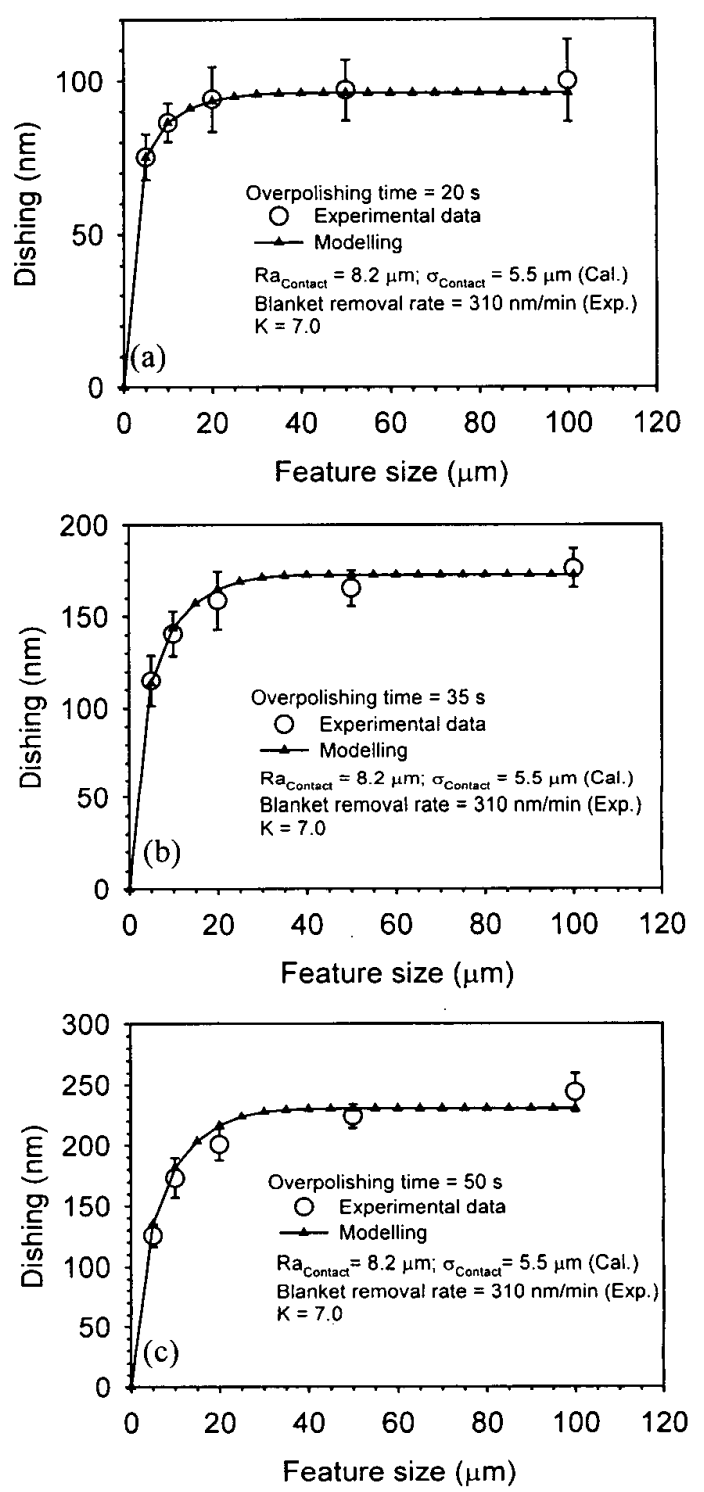

Fig. 10 Experimental data versus calculated data using the proposed model. 